\title{
CoRoT planet host stars
}

\author{
M. Fridlund ${ }^{1,2,3}$, G. Bruno ${ }^{4}$, M. Deleuil ${ }^{4}, \mathrm{D}$. Gandolfi ${ }^{5}$, and the CoRoT CEST team
}

\section{Introduction}

One topic that has taken on more and more importance in the field of exoplanetology during the period of the flight of the CoRoT and Kepler space missions, is the role of the host star. The fundamental parameters - those that can be measured directly - for an exoplanet are its radius and mass. For the host star, the fundamental parameters are its mass, radius, metallicity and age, and it is generally assumed that the two latter parameters are also applicable to associated planets. Obtaining these parameters allow the characterisation of the planet, the star and the system as such to take place. The two sets of parameters, planetary and stellar are intimately connected, since the planetary parameters depend on the measurement of the stellar values. The transit method measuring the radius of the planet and the radial velocity method obtaining the planetary (minimum) mass will only provide these parameters in terms of the stellar ones. As the launch of photometers into space has provided the opportunity for a photometric accuracy at the part-permillion (ppm) level and given that the almost simultaneous development of stabilised and well-calibrated ground based radial velocity measurements are reaching a level of accuracy better than $1 \mathrm{~m} \mathrm{~s}^{-1}$, it has become possible to begin to carry out high precision physics on our elusive exoplanetary targets. But the increasing precision in measurements of the planets parameters is not coupled to a similar increase in the measurements of the stellar parameters. For a direct measurement of the stellar masses and radii, we require that the star is either going to be very close (allowing interferometry to measure the diameter directly) and/or in an eclipsing binary system (Double-lined Eclipsing Spectroscopic Binary systems, usually designated SB2). The number of such objects is very low and those with (transiting) known exoplanets are even more rare. It was realised very early during the CoRoT project (when literally thousands of excellent light curves were being obtained), that good spectra of the large numbers of potential host stars were going to be required. As a consequence, large amounts of time were successfully applied for the ESO VLT/UVES combination, as well as later through a separate agreement with NASA on the Keck/HIRES instrument. This meant that modelling of the host stars spectra in order to determine the stellar parameters could begin. The process for how this was done is described in the individual papers relating to the discovery and confirmation of each CoRoT planet, as well as in Bruno (2015) and in Fridlund et al. (in prep.). Here we will briefly describe the methods and some of the conclusions reached. Later on during the flight of CoRoT, less and less VLT and Keck time were allocated to dedicated stellar spectra. But as time was passing the number of radial velocity spectra, obtained in order to determine the planetary masses, was building up and consequently so was the signal to noise $(\mathrm{S} / \mathrm{N})$ in the accumulated spectrum for each star. Therefore these radial velocity spectra (those obtained with the HARPS spectrograph have almost twice the resolution compared to the UVES/HIRES spectra) could be used for stellar modelling.

At the moment, the only way to determine stellar masses, radii and ages, is to obtain very high $S / N$ spectra of the star in question with an as high as possible spectral resolution $(R)$. Then one models the spectrum, fits the model to the data and, after (usually) having iterated the process a number of times, arrives at an effective temperature, a value for the local gravity at the surface, the abundances and the rotational velocity as well as the turbulent velocities. From these values and the light curve, one can estimate the stellar mass and then model where the star should be on its evolutionary track, and read of its radius and age. Although, this process appears pretty straightforward, there are a number of problems to be addressed. The first concerns the quality of the spectrum itself. A "high S/N" means larger 
than $S / N=100$ and is ideally better than $S / N=200$. For a spectrum with a resolution of $R=60000$ this means that typically one night is required on an $8 \mathrm{~m}$ class telescope for a star in the brighter half of the CoRoT magnitude range of $12-16$ in order to obtain a $S / N>100$. For the Kepler stars that are typically 3 magn brighter, you can obtain spectra of two such stars per night with a $10 \mathrm{~m}$ class telescope, but on the other hand you have many more bright targets because of the larger field of the Kepler telescope. In order to follow-up a planet candidate, one needs ultimately to obtain the radial velocity signature of the planet, and there is a direct relation between the apparent brightness of the star and the precision with which one can measure the radial velocity amplitude. It means that the smaller the planet is the brighter the star has to be, in order to confirm the nature and then derive the planets mass. A consequence of this was that a large number of potential planet candidates observed with CoRoT that had host stars fainter than about magnitude 15 could not be followed up at the time, and had to be postponed to an uncertain future (e.g. observations with the CARMENES and ESPRESSO spectrographs). To accurately measuring the fundamental stellar parameters (and properly understand stars in general), the way forward is going to be dependent on another major result from space. First obtained with CoRoT and then confirmed and enhanced with NASA's Kepler mission, it was demonstrated that the accuracy commensurate with the precision of the stellar fundamental parameter measurements could be achieved using asteroseismology. Since space observations are required for bulk measurements, this is now, since the selection in March 2014 of PLATO (Rauer et al. 2014) as ESA's M3 mission on the horizon. Unfortunately, the launch of PLATO will still be about one decade into the future, and we have literally thousands of light curves from CoRoT and Kepler, as well as thousands of high precision radial velocity curves and associated data awaiting further interpretation. It is therefore needed to determine the fundamental physical parameters of exoplanetary host stars as well as it is currently possible with the available methods.

\section{Required stellar parameters}

In order to correctly analyze the exoplanet parameters, the most important stellar parameters that are required are the mass and radii. Acquiring those makes it possible to estimate the average density, with the errors in the planetary parameters depending only on the errors in the actual measurements, and, the errors in the estimation of the stellar parameters. And here lie the crux. It can be shown (e.g. Rauer et al. 2014), that in order to be able to classify planets and draw reasonable conclusions from the bulk densities, one needs to determine the planetary radii to a precision of $2 \%$ and the masses to definitely better than $10 \%$ and preferably better than $5 \%$. It is sometimes assumed that we know the mass-luminosity relation, both from measurements of Double-lined Eclipsing Spectroscopic Binary systems (SB2), and from theoretical modeling, well enough. The problem is that most stars searched for planets, either with radial velocities or with transits, are low-mass, low-luminosity objects. And the scatter on the empirical mass-luminosity relation is greatest for stars of masses less than one solar mass. Which is of course the great majority.
This scatter is probably, in part, due to age effects in the star, as it gets brighter already on the main sequence. The Sun has brightened by an estimated $30 \%$ so far during its main sequence life and will brighten by more during the rest of its main sequence phase of almost $10^{10}$ years, while it only loses a negligible amount of mass through the solar wind during the same time. The solution that has been put forward is to use the new method of asteroseismology (developed to a large extent due to the observations of CoRoT). By observing the power spectrum of acoustically induced variations in the light output of the star, it is possible to determine the current mass and radii of the star with the required precision. This has been amply demonstrated with the CoRoT and Kepler space missions, but unfortunately the technology for making observations of the power spectrum of exoplanetary host stars in bulk is still, about 10 years away with the launch of the PLATO spacecraft. Instead we are required to analyze the optical spectrum of the host star with the highest possible precision. But it is not enough to determine the mass and radii. The rotational velocity of the star, as well as the macro- and micro-turbulent velocities, is important - particularly in the context of the activity and age of the star. And the age of the star is of course required when we want to say something about the evolutionary state of the planetary system in question. As an example, one can point out that the distribution of planets in most systems requires the age of the system in order to account for migration or the stability of the orbits. In order to study the star with available tools, there are essentially only two: photometry and spectroscopy. Using a photometric method is in practice the same as obtaining a very low-resolution spectrum. Photometry depends on introducing carefully designed and measured filters into the ray path which would allow only specific parts of the stellar spectrum to impinge on the detector. The flux through different filters can be subtracted from each other to create color indexes, which can be shown to depend on certain of the fundamental stellar parameters such as $T_{\text {eff }}, \log g$, the interstellar extinction and the metallicity. It is a very coarse method, with large individual errors and best useful for evaluating large statistical materials or for creating e.g. input catalogues. Spectroscopy provides very high resolution and detail. There are literally millions of atomic and molecular lines superposed on the continuum, as well as the shape of the continuum, that can be used to determine the fundamental parameters, including the stellar velocities. The drawback is that one achieves the best results when one is observing individual objects, with large telescopes, for a long time. Fiber-fed spectrographs can be made multi-tasking (i.e. a large number of fibres can be feeding the same spectrograph simultaneously), but then the resolution will be limited due to the finite size of the detector.

\section{Analysing the spectrum}

There are several ways and means of analyzing the optical spectrum of an exoplanetary host star, but they all have in common that one has to start with a high quality $(\mathrm{S} / \mathrm{N}$ and spectral resolution) spectrum. From this we can discern the stellar velocities, both the projected rotational and turbulent velocities, the effective temperature, $\log g$, the $[\mathrm{Fe} / \mathrm{H}]$, and other abundances. From the transiting light 
curve, we also get an independent estimate of the stellar density and $\log g$. From these parameters we can derive the masses and radii and put the star onto model evolutionary tracks and estimate the age, albeit with very large error bars. We also get an estimate of the stellar activity (which can also be used under certain circumstances as an age index). The presence of e.g. Lithium in the spectrum can either indicate youth, or in hotter solar type stars be a way to determine the exact spectral class. As mentioned above, the idea is to take the observed spectrum, normalize it and then compare it to our models and eventually find the model that is most likely to represent reality. There is a large amount of parameters to fit, which on the one hand make the process complex, but on the other hand make it more robust in order to find a unique solution. One method is to use the equivalent width of metal lines. Three parameters are usually used as input to the process: the equivalent width (EW), the excitation potential, and the abundance of the metal in question. The EW is found from the spectrum, the excitation potential from atomic data and the abundance is derived through curve of growth analysis. Different atmospheric parameters produce different curves of growth and, by analyzing a large amount of different metal lines and excitation parameters, one can fit the abundance. This is the procedure used by the Versatile Wavelength Analysis (VWA) package (Bruntt et al. 2010), and which has automated the process and has been used for a number of CoRoT stars. One needs to start with the normalized spectrum and select several hundred (ideally) weak, unblended metal lines. Using a first guess of $T_{\text {eff }}, \log g,[\mathrm{Fe} / \mathrm{H}]$ and the velocities $V_{\text {micro }}, V_{\text {macro }}$ and $v \sin i$, a synthetic spectrum is calculated, the equivalent width of the selected metal lines are calculated and fitted to the observed spectrum. After visual inspection and the discarding of complicated lines, a series of models is iteratively calculated and the results are minimized with respect to the observed spectrum. The other main approach is by fitting the whole spectrum, thus the actual shape of the calculated spectra to the observed and normalized spectrum. This is the method used by Spectroscopy Made Easy (SME; Valenti \& Piskunov, 1996). SME calculates the spectral model using a grid of models (Kurucz 2005 or MARCS, Gustafsson et al. 2008). The SME utilizes automatic parameter optimization using a Levenberg-Marquardt minimization algorithm. Synthetic spectra are calculated on the fly by a built in spectrum synthesis code, for a set of global model parameters and specified spectral line data. Starting from the user-provided initial values, synthetic spectra are computed for small offsets in different directions for a subset of parameters defined to be "free". The model atmospheres required for this are calculated through interpolating the grid of models mentioned above. SME allows one to choose which parameters to fit or to fix, or to solve for all the atmospheric parameters at the same time. Normally, one begins by separately fitting the wings of the profiles of one or more of the Balmer lines, and solving for $T_{\text {eff }}$. The next step is to get an idea about the abundances, particularly $[\mathrm{Fe} / \mathrm{H}]$ and the metal lines used for the determination of $\log g$. Keeping the $T_{\text {eff }}$ estimate and the abundances as fixed parameters, we then fit the spectrum of $\log g$ sensitive lines, especially the pressure-sensitive strong metal lines such as CaI at $6122 \AA, 6162 \AA$ And $6438 \AA$, the $\mathrm{Mg}$ Ib triplet at 5160-5185 $\AA \AA$, and/or the NaI D lines. After several fits with varying starting values, the standard deviation of the results gives the internal uncertainties on the derived parameters. Fixing some parameters, moreover, is helpful when the spectrum has a low $S / N$ ratio. It has also been possible to determine the fundamental parameters of stars with large $v \sin i$ with SME such as e.g. CoRoT-11 (Gandolfi et al. 2010) that has a $v \sin i$ of $40 \mathrm{~km} \mathrm{~s}^{-1}$. Further, we refer to Bruno (2015), Fridlund et al. (in prep.) and the individual CoRoT-papers for detail of the process of determining the stellar parameters. Here we, finally, only stress the two main problems with the spectral analysis.

\section{The two problems}

There are two major problems with both of these methods (VWA and SME). Both require spectra with high $S / N$ to work well. This is especially true for the determination of $\log g$ where VWA requires the identification of a very large number of weak metal lines at high S/N, while SME has the problem of there being very few pressure sensitive metal lines found in the available parts of the spectrum. Furthermore, several of those lines, such as the MgI triplet, are in regions where the continuum is depressed by large number of metal lines, making any determination of the $\log$ $g$ based on them uncertain. The calculated value of $\log g$ is very sensitive both to $T_{\text {eff }}$ and to the abundance of the particular metal. It is possible, in the individual case to determine $\log g$ to \pm 0.1 dex but a more realistic $1 \sigma$ error is \pm 0.2 dex. The $T_{\text {eff }}$ is easily determined for solar type star with temperatures between $4500 \mathrm{~K}$ and $7000 \mathrm{~K}$, especially by fitting the wings of the $\mathrm{H} \alpha$ Balmer line $(\mathrm{H} \beta$ has depressed wings due to a large amount of metal lines and $\mathrm{H} \gamma$ is usually not present in the Echelle spectra obtained with the spectrographs in question). The $\mathrm{H} \alpha$ line is usually found at or close to the edge of the Echelle order in the spectrographs used such as UVES, SOPHIE and HARPS. This is of course because $\mathrm{H} \alpha$ is uninteresting when determining radial velocities. Having $\mathrm{H} \alpha$ with its extremely broad (for $T_{\text {eff }}>5000 \mathrm{~K}$ ) wings make it difficult to determine the continuum exactly - particularly if the spectrum have poor S/N. Such errors can manifest themselves as errors in Teff of $\pm 200 \mathrm{~K}$ or even larger. This has a strong impact on the determination of particularly the value of $\log g$ (e.g. Fuhrmann et al. 2011). There are exceptions. The FIES spectrograph at the Nordic Optical Telescope in La Palma have the $\mathrm{H} \alpha$ line well centered in the order where it is found, and tests have shown that in a well/exposed spectrum of a G0V star one can determine $T_{\text {eff }}$ with a measurement error of $\pm 70 \mathrm{~K}$. In Table III.4.1 we present the $T_{\text {eff }}$ as determined with several methods from very good (high $S / N$ ). It is clear that with such good material different methods give a good result. The original observations that are somewhat different were obtained using only one model instead of a complete grid of different models on which to iterate to a minimum fitting. One must always remember that the errors in this modelling does not include the errors due to the imperfections and assumptions present within the models themselves.

If one has a material obtained with a spectrograph that provides less accurate $T_{\text {eff }}$, it may be profitable to initiate a separate program for the determination of the effective temperature alone using a suitable spectrograph (preferable a long slit spectrograph with representative resolution) that allows a good determination of this crucial parameter. 
The CoRoT Legacy Book

Table III.4.1. Effective temperature derivation for the first 9 CoRoT host stars. $T_{\text {eff }}$ is derived using SME 4.43 with ATLAS12 models, SME 4.43 with MARCS models, the $V W A$ method with MARCS models, and the $T_{\text {eff }}$ from the original discovery paper.

\begin{tabular}{lccccc}
\hline \hline \multirow{2}{*}{ Target } & $\begin{array}{c}T_{\text {eff }}(\mathrm{K}) \\
\text { ATLAS12 }\end{array}$ & $\begin{array}{c}T_{\text {eff }}(\mathrm{K}) \\
\text { MARCS }\end{array}$ & $\begin{array}{c}T_{\text {eff }}(\mathrm{K}) \\
\text { VWA ion eq. }\end{array}$ & $T_{\text {eff }}$ (orig.) & Orig. Source \\
\hline CoRoT-1 & $6145 \pm 75$ & $6075 \pm 75$ & $6090 \pm 80$ & $5950 \pm 150$ & Barge et al. (2008) \\
CoRoT-2 & $5330 \pm 75$ & $5300 \pm 75$ & $5580 \pm 80$ & $5450 \pm 120$ & Alonso et al. (2008) \\
CoRoT-3 & $6575 \pm 100$ & $6575 \pm 100$ & $6600 \pm 40$ & $6740 \pm 140$ & Deleuil et al. (2008) \\
CoRoT-4 & $5975 \pm 100$ & $5975 \pm 100$ & $6090 \pm 50$ & $6190 \pm 60$ & Moutou et al. (2008) \\
CoRoT-5 & $6065 \pm 100$ & $5980 \pm 100$ & $6030 \pm 50$ & $6100 \pm 65$ & Rauer et al. (2009) \\
CoRoT-6 & $6025 \pm 100$ & $6025 \pm 100$ & $6060 \pm 50$ & $6090 \pm 70$ & Fridlund et al. (2010) \\
CoRoT-7 & $5290 \pm 44$ & - & $5297 \pm 13$ & $5275 \pm 75$ & Léger et al. (2009) \\
CoRoT-8 & $4950 \pm 100$ & $5095 \pm 100$ & $5050 \pm 50$ & $5080 \pm 80$ & Bordé et al. (2010) \\
CoRoT-9 & $5575 \pm 100$ & $5575 \pm 100$ & $5580 \pm 70$ & $5625 \pm 80$ & Deeg et al. (2010) \\
\hline
\end{tabular}

\section{A future evolution beyond CoRoT}

As stated in the previous section, their are several problems with determining the fundamental stellar parameters from the spectrum of the host star, resulting in the physical parameters of the planet $(\mathrm{s})$ having uncertainties large enough to obstruct comparisons with theoretical models. Some relief is expected from the Gaia catalogue to emerge in a few years since its improved distances for essentially all CoRoT and Kepler stars will provide a better value for $T_{\text {eff }}$. Nevertheless, the mass and radius of any host star not located in a suitable binary system will rely on evolutionary models. The age of the system will remain uncertain to a very high degree.

The solution to this and the methods that will allow exoplanetology turning into a true comparative science allowing comparisons between different (types of) exosystems and even with our own Solar System - is to implement asteroseismology systematically. As has been proven unambiguously - first by CoRoT and then by Kepler asteroseismology can provide information about the host star that will allow ultimately the determination of the stellar host parameters to the required precision of a few percent (in the case of age maybe 10 or a few tens of percent), allowing exoplanet geology and atmospheric research to make the transition into detailed and individual science. All that is needed is the long and uninterrupted high precision photometric light curve of the host star in question. This is the basis for the PLATO M3 mission of ESA to be launched towards the middle of the next decade. For brighter stars (magnitude brighter than 11.5) reversing the asterseismological parameters will then give very high precision of, among other things, the stellar mass, radius and age.

When PLATO eventually flies, and very likely changes not only exoplanetology but also stellar physics and everything relating to these two topics, it should be recalled that this work started with the CoRoT mission. The little spacecraft that could do its job.

Acknowledgements. MF is grateful to MPIA in Heidelberg and to T. Henning who provided a research grant allowing him to stay in Heidelberg during 2015.

\section{References}

Alonso, R., Auvergne, M., Baglin, A., et al. 2008, A\&A, 482, L21

Barge, P., Baglin, A., Auvergne, M., et al. 2008, A\&A, 482, L17

Bonomo, A. S., Santerne, A., Alonso, R., et al. 2010, A\&A, 520, A65

Bordé, P., Bouchy, F., Deleuil, M., et al. 2010, A\&A, 520, A66

Bouchy, F., Deleuil, M., Guillot, T., et al. 2011, A\&A, 525, A68

Bruno, G. 2015, Ph.D. Theses, LAM

Bruntt, H., Bedding, T. R., Quirion, P.-O., et al. 2010, MNRAS, 405, 1907

Cabrera, J., Bruntt, H., Ollivier, M., et al. 2010, A\&A, 522, A110

Deeg, H. J., Moutou, C., Erikson, A., et al. 2010, Nature, 464,384

Deleuil, M., Deeg, H.,J., Alonso, R., et al. 2008, A\&A, 491, 889

Fridlund, M., Hebrard, G., Alonso, R., et al. 2010, A\&A, 512, A14

Fuhrmann, K., Chini, R., Hoffmeister, V. H., et al. 2011, MNRAS, 411, 2311

Gandolfi, D., Hebrard, G., Alonso, R., et al. 2010, A\&A, 524, A55

Gillon, M., Hatzes, A., Csizmadia, Sz., et al. 2010, A\&A, 520, A97

Gustafsson, B., Edvardsson, B., Eriksson, K., et al. 2008, A\&A, 486, 951

Kurucz, R. L., 2005, MSAIS, 8, 14

Léger, A., Rouan, D., Schneider, J., et al. 2009, A\&A, 506, 287

Moutou, C., Bruntt, H., Guillot, T., et al. 2008, A\&A, 488, L47

Rauer, H., Queloz, D., Csizmadia, Sz., et al. 2009, A\&A, 506, 281

Rauer, H., Catala, C., Aerts, C., et al. 2014, ExA, 38, 249

Tingley, B., Endl, M., Gazzano, J.-C., et al. 2011, A\&A, 528, 97

Valenti, J. A., Piskunov, N., et al. 1996, A\&AS, 118, 595 